\title{
Interactive Modeling of 3D Tree with Ball B-Spline Curves
}

\author{
Zhongke Wu, Mingquan Zhou and Xingce Wang
}

\author{
College of Information Science and Technology \\ Beijing Normal University \\ Beijing 100875, China
}

\begin{abstract}
A novel approach to modeling realistic tree easily through interactive methods based on ball B-Spline Curves (BBSCs) and an efficient graph based data structure of tree model is proposed in the paper. As BBSCs are flexible for modifying, deforming and editing, these methods provide intuitive interaction and more freedom for users to model trees. If conjuncted with other methods like generating tree models through L-systems or iterated function systems (IFS), the models are more realistic and natural through modifying and editing. The method can be applied to the design of bonsai tree models.
\end{abstract}

Keywords-Virtual plants, trees modeling, Ball B-Spline Curve, 3D Interactive modeling

\section{INTRODUCTION}

Many contributions have been made to plant modeling. L-system [4], Iterated Function Systems (IFS) [8-10], particle systems [21], Automata [3], stochastic matrix (ramification matrix) [31] and image based method [1] are used to describe topological structures in plants. For the faithfulness of the models to botanical nature of trees and plants, Philippe de Reffye proposed a model through simulating the growth of plants [32] and Oppenheimer used random number in fractal modeling to generate plants in real time [30]. Among these methods, L-system is the mainstream method. Because of the structural complexity and freeform shapes of trees in nature, the models generated by L-system or other fractal methods are not plentiful enough. Therefore interactive method is becoming a hot topic in modeling plants. Bernd Lintermann and Oliver Deussen introduce interactive method of modeling plants in 1999 [12]; Later Makoto Okabe, etc. proposes a 2D freehand sketch method to design trees [13]; furthermore, Lisa Streit, etc. describes a 3D sketch method to model plants [14]; Xing Zhao, etc. investigates interactive simulation of plant architecture based on a dual scale automation model [15]; Xi Wang, etc. focuses on interactive modeling tree bark [16].

In geometric representations of plants, a variety of methods for representing 3D objects are used. In boundary representation methods, polygon mesh [6] and subdivision

Manuscript Received on 30 June 2008

E-Mail: mqzhou@bnu.edu.cn surfaces [17], Cone-Sphere [18], generalized cylinder [2], implicit methods [19] like Blobtree [5, 22] and convolution surfaces [20] are discussed. Even volume model [3] is also explored to represent plants.

This paper proposes a novel approach to modeling trees with ball B-Spline curves [7, 11] easily through interactive methods. BBSC is one kind of skeleton based representation method of 3D solid tubular object. Recently skeleton based representation is hot topic. Sederberg and Farouki proposed interval B-Splines [24] by using 2 intervals to define rectangular regions instead of using simple control points. Further Lin and Rokne [25] introduced disk/ball Bézier curve/surface by using disk or ball instead of control points. Randriambelosoa tried to extend this to B-Spline in constructing $G^{1}$ ball B-Spline by connecting 2 ball Bézier curves. But the conditions are too complex even for some special cases [26]. For general case it is very difficult if not impossible. Generalized cylinders [27-29] are one kind of skeleton based representations, which are defined by moving a 2D contour along a 3D trajectory, i.e. generalized cylinder is defined through its skeleton and Frenet frame on skeleton. The tangent and normal vector are used in representation formula. So it is a constructive or procedural representation method.

BBSC is a new representation of tubular objects with thickness variations through directly defining the centreline (skeleton) of solid regions as well as their radii in B-Spline form, not constructing by combining ball Bézier curves. Comparing with generalized cylinder, BBSC is defined by one parametric equation, i.e. B-spline form. Therefore, the explicit representation of ball B-spline is flexible for transformation, manipulation, deformation, etc. The operations can be implemented through changing control points and radii. As the representation of generalized cylinder dependents on the tangent and normal vector at each skeleton point, these operations are not intuitive. Moreover, the properties of BBSC can be explored directly and globally. But the properties' investigation in generalized cylinder must dependent on each local point's tangent and normal.

Therefore, BBSC has good properties and is very suitable for interactively generating trees. The paper is organized as follows. In section 2, the representation method of a tree based on ball B-Splines is discussed; In section 3, various interactive modeling methods based above representation model are described; In the section 4, local editing function are investigated; In final section, some results and examples are 
shown.

\section{TREE REPRESENTATION}

\subsection{Topology structure}

An efficient graph based data structure (tree data structure) of tree model is shown in Fig. 1.The data structure is built to represent the structural relationships among stems of trees. A general tree data structure is used to cover various trees' branch structures,. For efficient searching and modifying, the two direction pointers between parent and children are necessary. In each node of the tree data structure, the topological information of its parent and children and its geometric representation based on BBSCs are stored. For example, when modifying a branch's geometry information, to keep the continuity, its parent and children node information are needed.

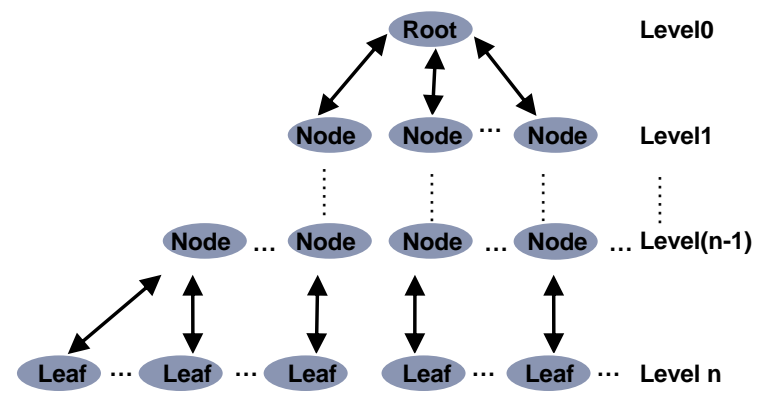

Fig. 1 Hierarchical Structure

After the topological model is built, the relationship among these stems can be easily found and the efficient management and manipulation can be available. And it can also provide great potential of interactively creating realistic tree models easily.

\subsection{Ball B-Spline Curve [7, 11]}

Let $N_{i, p}(t)$ be the i-th B-Spline basis of degree $\mathrm{p}$ with knot vector $\left[u_{0}, \ldots, u_{m}\right]=\left\{a, \ldots a, u_{p+1}, \ldots, u_{m-p-1}, b \ldots, b\right\}$.Here, $<P_{i} ; r_{i}>$ is a ball centered at $P_{i}$ with radius $r_{i}$.

The Ball B-Spline Curve (BBSC) is therefore defined as $<B>(t)=\sum_{i=0}^{n} N_{i, p}(t)<P_{i} ; r_{i}>$, where $P_{i}$ is control point and $r_{i}$ is control radius.

$$
\text { As: }<B>(t)=\sum_{i=0}^{n} N_{i, p}(t)<P_{i} ; r_{i}>=<\sum_{i=0}^{n} N_{i, p}(t) P_{i} ; \sum_{i=0}^{n} N_{i, p}(t) r_{i}>\text {, a }
$$

Ball B-Spline curve can be regard as two parts: a 3D B-Spline curve, i.e. the center curve (or skeleton): $\mathrm{c}(\mathrm{t})=\sum_{i=0}^{n} N_{i, p}(t) P_{i}$, and a B-Spline scalar function, i.e. the radius function $\mathrm{r}(\mathrm{t})=\sum_{i=0}^{n} N_{i, p}(t) r_{i}$. Therefore most properties and algorithms can be obtained by applying B-Spline curve and function to the two parts of BBSC respectively. Owing to the perfect symmetry property of balls, the curve $c(t)$ constructed from the centers of balls is exactly the center curve of the $3 \mathrm{D}$ region represented by the Ball B-Spline curve. The difference between B-Spline curve and BBSC is shown in Fig. 2.

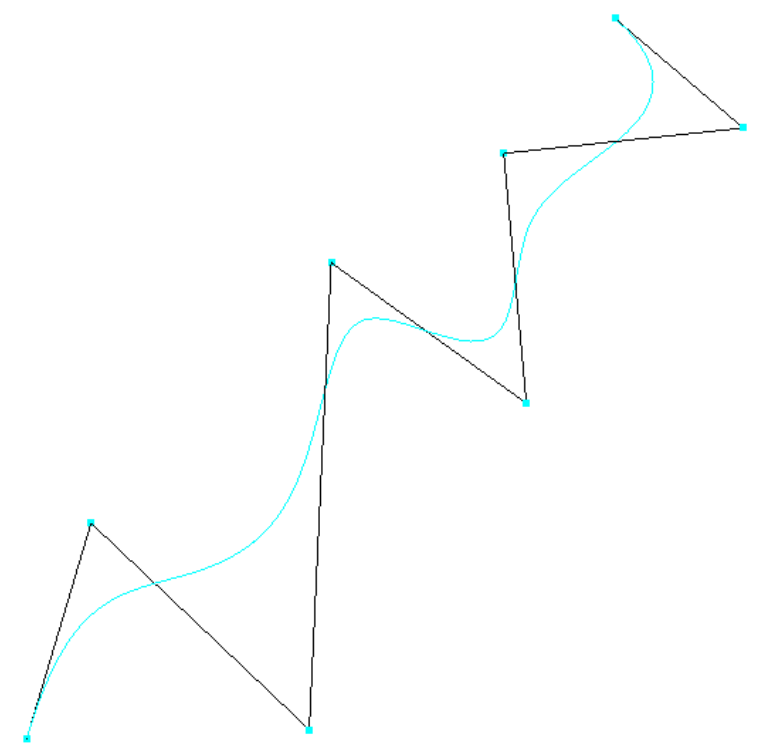

(a) Spatial curve represented by B-Spline curve

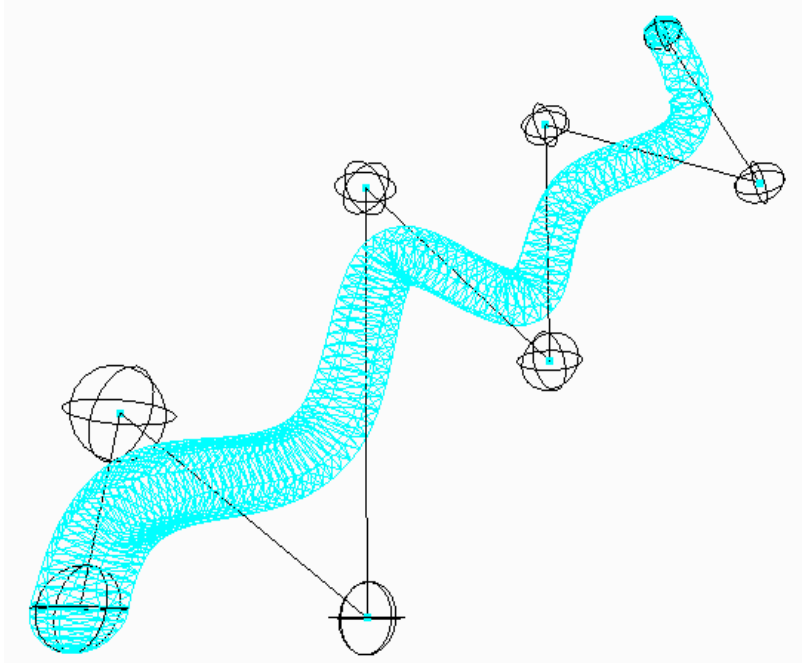

(b) 3D solid region represented by BBSC

Fig. 2 Comparison of B-Spline curve and BBSC

From the paper [7], it can be seen that BBSC has solid mathematical fundamentals. It can represent centerlines and every point of 3D solid objects. It inherits theses properties from B-Splines, like precise and efficient evaluation. It is flexible for manipulation, deformation and morphing. It is a parametric representation and thus requires small dataset for representing 
freeform shapes. These properties provide great potentials to build a flexible plant model interactively.

Similar with B-Spline modeling, a ball B-Spline curve can be generated through interpolating and approximating as shown in Fig. 3. We can modify the 3D shape by deforming ball B-Spline curves through modifying its control points and radii as shown in Fig. 4.

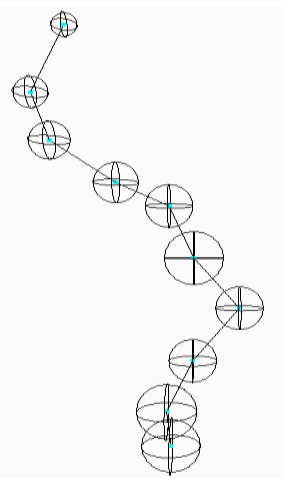

(a) Points and radii

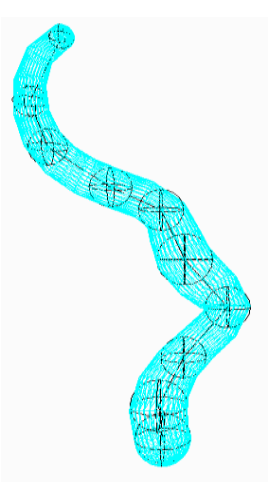

(b) Interpolation Fig. 3 Interpolation and approximation of BBSC

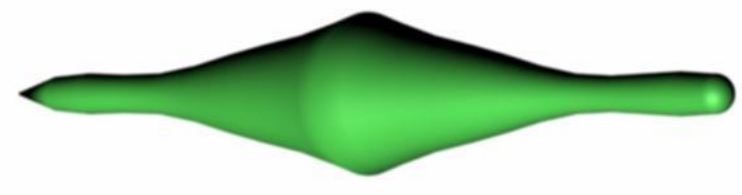

(a) Original Object

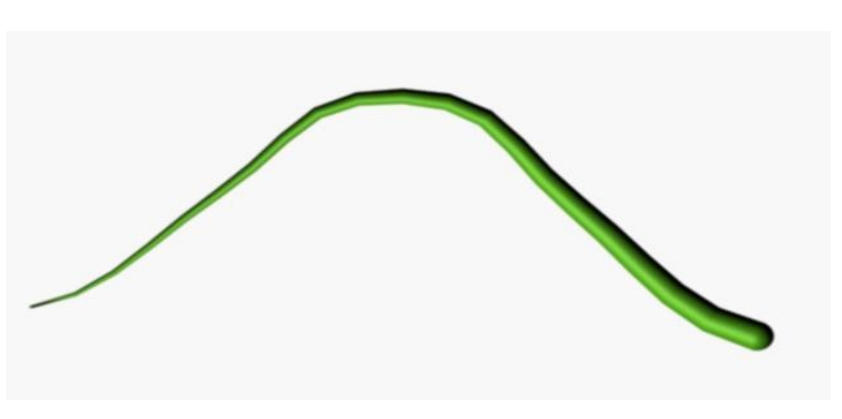

(b) Deformed Object

Fig. 4 Deformation of BBSC through changing its control points and radii

Further properties and algorithms about BBSC, readers can refer to the paper [7].

\subsection{Representing tree with BBSCs}

For geometric data, a BBSC is used to represent each stem of a tree shown in Fig.5.

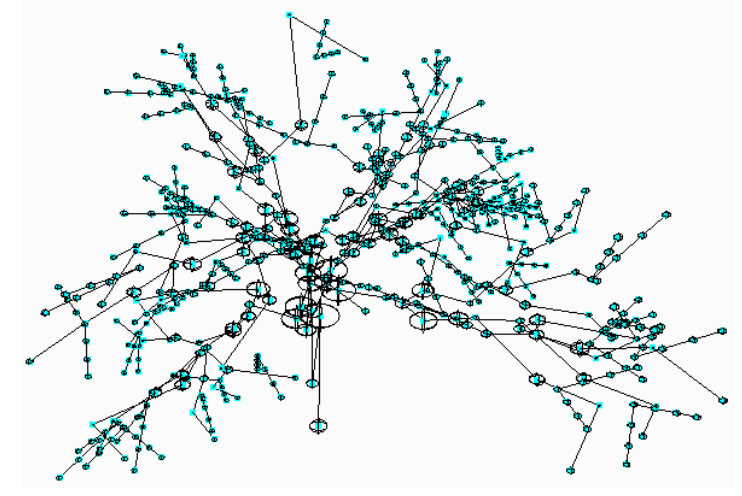

Fig. 5 Tree represented by ball B-Spline curves (Control balls are shown)

\section{INTERACTIVE MODELING METHODS}

\subsection{Interactively creating tree models}

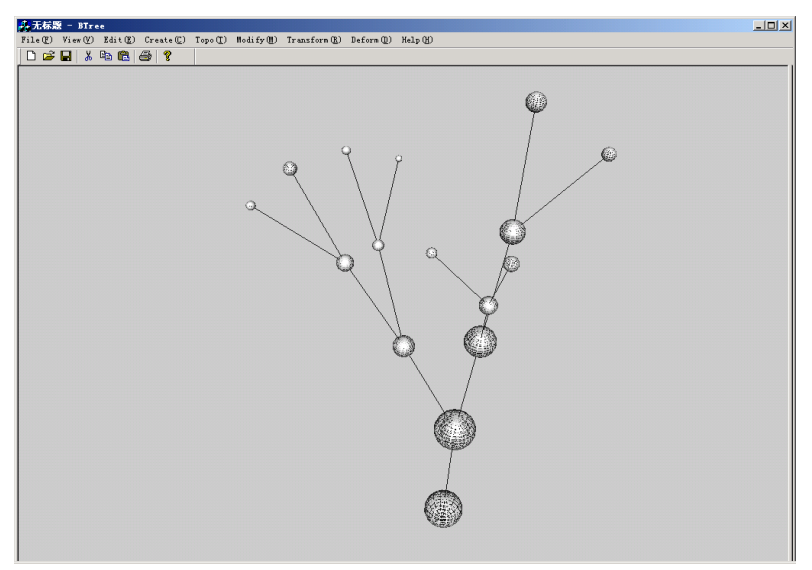

(a) Interactively inputting a group of balls with variant radii in 3D space

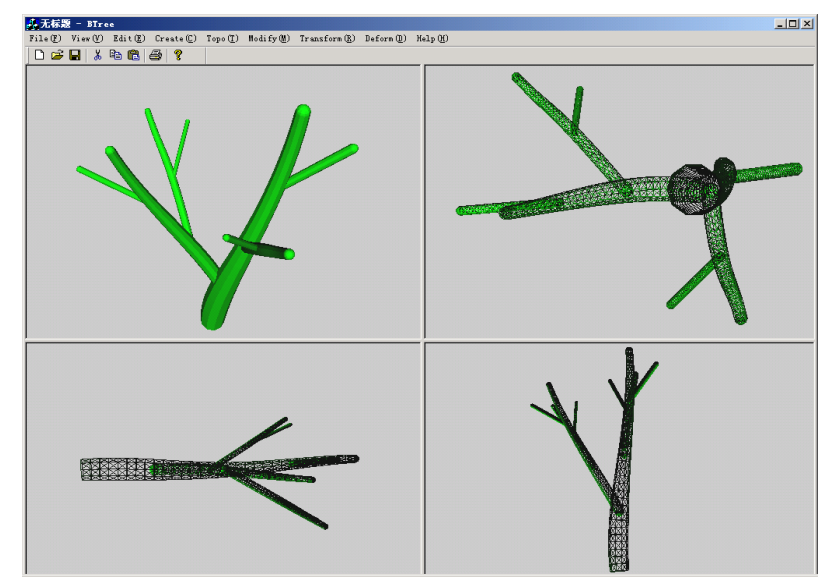

(b) Generating tree model

Fig. 7 Interactive creation of tree model

A tree can be created by rolling a radius-varying ball shown in Fig. 7. During rolling balls, a sequence of position and radii data can be obtained. By interpolating or approximating these points and radii, a BBSC is generated to represent a stem. A tree model is generated by iterating the above procedures. 


\subsection{Deforming methods}
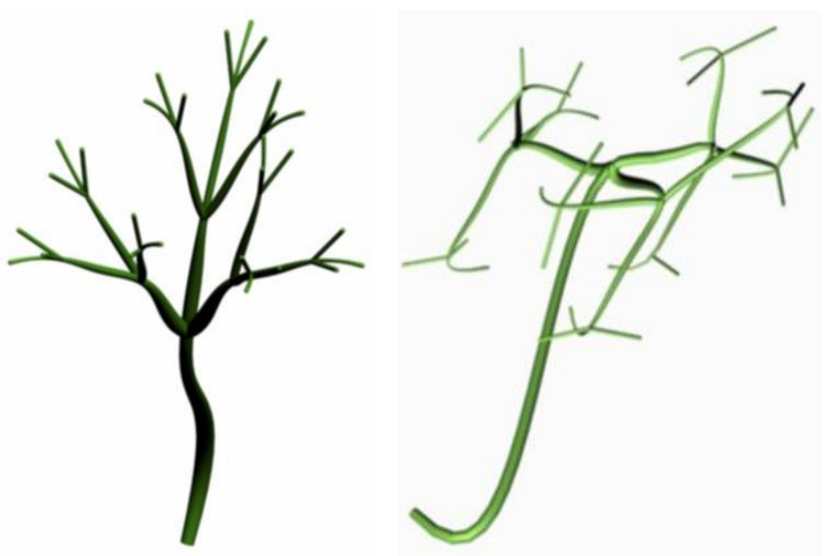

(a) Original model Fig. 8 Global and local deforming methods

Global and local deforming methods of tree models are implemented through exerting the deformation to its geometric model of BBSCs. Global deforming includes shearing, bending, twisting, stretching, taper, and their compound, etc. These deformations are applied to the whole tree, whole branch or whole stem. Local deforming is to deform some local part of some stem. For these two kinds of deformation methods, they are implemented through deforming its geometric representation, i.e. this deformation is implemented through deforming BBSCs as mentioned above. Fig. 8 shows the result after global and local deformation.

\subsection{Morphing method}

Morphing is a method of generating a sequence of shapes between given two or more than two different shapes. By given two or more tree model, these metamorphosis tree models can be created by simulating their morphing procedure. The algorithm for given 2 tree models case is explained, for more than 2 tree models case, the algorithm is similar.

(1) The corresponding stems between given initial and final tree models is found or indicated.

(2) For each couple of corresponding stems, metamorphosis stem is generated. As for each stem, its BBSC representation can be obtained. Therefore the problem converts into generating metamorphosis between two or more than two BBSCs.

(3) Now the generation method of metamorphosis BBSCs when given two or more than two BBSCs is discussed here. For clearer explanation of interpolating two BBSCs to generate metamorphosis BBSCs, it can be assumed that the two BBSCs have the same degree, number of control points and knot vector, that is

$$
<B_{1}>(t)=\sum_{i=0}^{n} N_{i, p}(t)<P_{i} ; r_{i}>
$$

Here, $<B_{2}>(t)=\sum_{i=0}^{n} N_{i, p}(t)<P_{i} ; r_{i}>$ and $\mathrm{T}$ is the knot vector. Therefore for the given two BBSCs case, the metamorphosis BBSCs can be regard as linear interpolation between the given 2 BBSCs, i.e. the metamorphosis BBSCs can be generated by interpolating $\left\langle B_{1}\right\rangle$ and $\left\langle B_{2}\right\rangle$ 's corresponding control points and radii. In this paper only linear interpolation is discussed. Suppose $\mathrm{m}+1$ metamorphosis BBSCs is given, the $m+1$ curve has the same degree, control points number and knot vector $\langle\mathrm{T}\rangle$. The control points of these BBSCs are $P_{j}^{i}$ and control radii are $r_{j}^{i}$,then

$$
\left\{\begin{array}{c}
P_{j}^{i}=\left(1-\frac{(i+1)}{(m+2)}\right) * P_{j}+\frac{(i+1)}{(m+2)} * Q_{j} \\
r_{j}^{i}=\left(1-\frac{(i+1)}{(m+2)}\right) * r_{j}+\frac{(i+1)}{(m+2)} * s_{j}
\end{array}\right.
$$

where $j=0,1, \ldots \ldots ., n$. When two BBSCs have different degree, the low degree curve can be converted to the higher degree through elevation algorithm. For two BBSCs with different control point number and knot vector, they can be converted to two BBSCs with same number of control points and knot vector through inserting knots. The algorithm is similar to B-Spline curve. So it needn't to explain in detail. For giving more than two tree models, B-spline curve and scalar function interpolation can be applied to the corresponding control points and radii of these BBSCs to generate the metamorphosis BBSCs.

(4) After generating all metamorphosis stems, these stems can be combined to generate metamorphosis tree model according to the metamorphosis order. So the morphing procedure is simulated as shown in Fig. 9

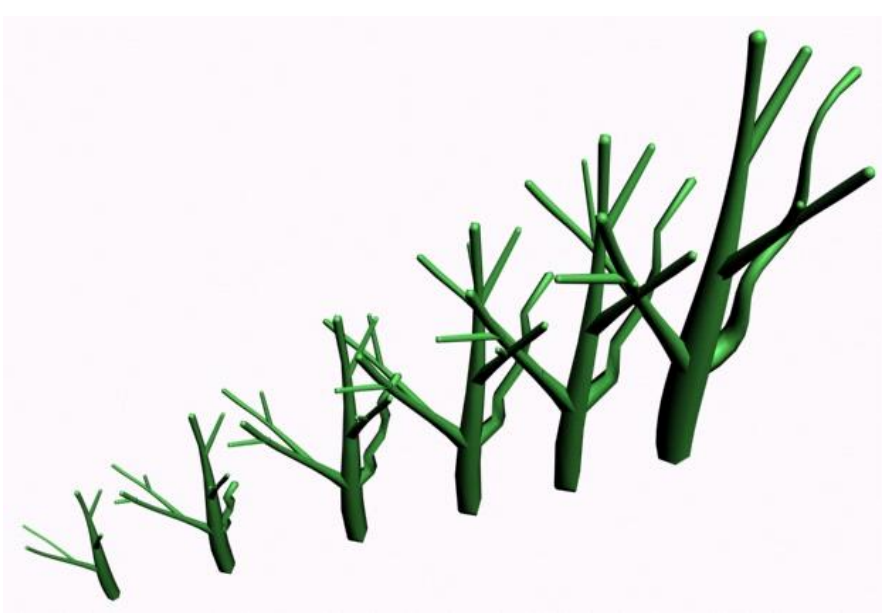

Fig. 9 Morphing between two trees with different topology and geometry 


\subsection{Modeling by combining branches}

Combining simple components (branches), a complicated tree can be modeled which is shown in Fig. 10. During the combination, some necessary transformations are used. Currently theses branches are only put together without any blending operations.
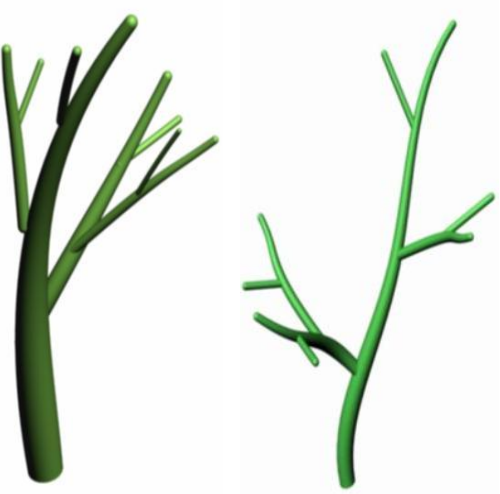

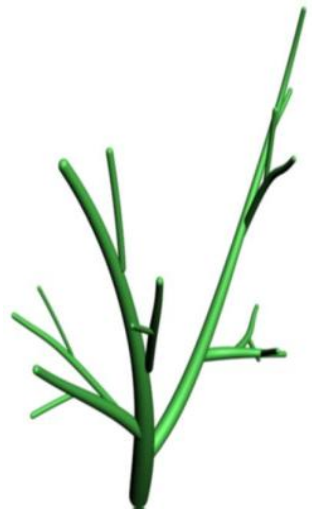
(b) Branch B
(c) Combined result
(a) Branch A
Fig. 10 Combining branches to generate a complex tree

\section{INTERACTIVELY EDITING METHODS}

After obtaining a tree model through interaction or procedural methods like L-system, some editing methods are used to refine its topology and geometry of the model as shown in Fig. 11. A branch is pruned and the shape of a stem is deformed through changing its control points and radii locally. For each stem, we can modify its position, direction globally as well as change its local geometry through modifying its geometry representation---BBSCs. As the topological data structure, we can exert these editing methods to only one stem or the branch starting from the stem.

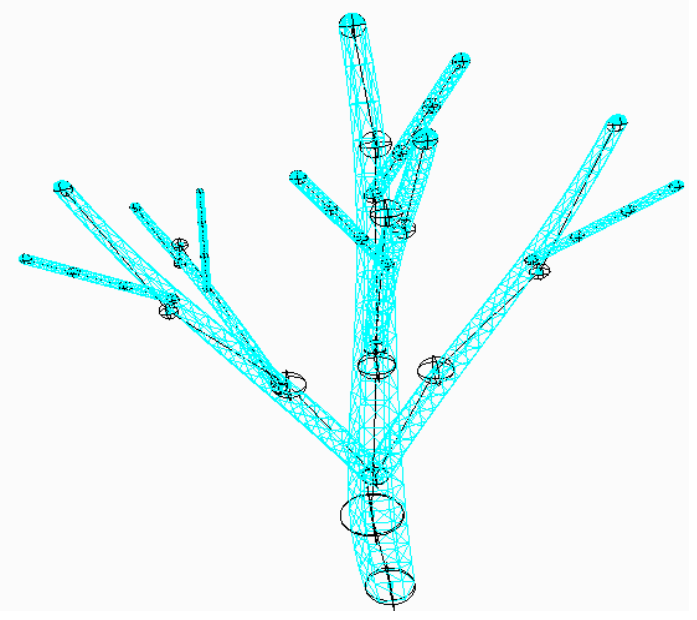

(a) Original tree model

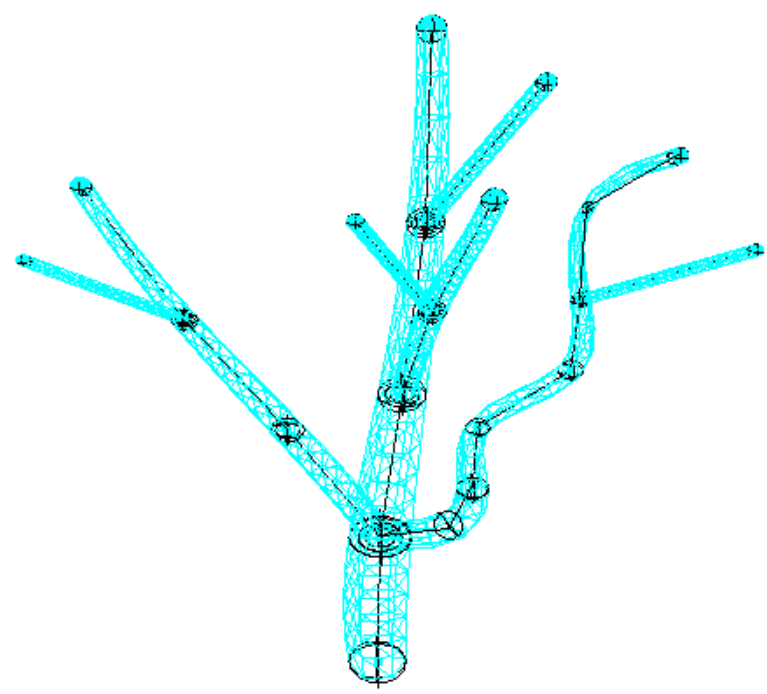

(b) Tree model after pruning and editing

Fig.11 Interactively editing of topological and geometric data

\section{RESULTS}

A general idea of modelling plant with BBSCs through interaction is proposed. As BBSCs are defined in B-Spline forms, the evaluation, tessellation and manipulation are as fast as B-Spline curves. Rendering of these plant models are implemented trough rendering the triangle mesh after tessellations. So the technique can be applied to interactively modeling plants in real time. Complex structure and freeform shape of a tree can be generated through interaction. A variety of interactively modeling methods are investigated which provide more intuitive interaction and freedom for user to create trees and assist users for creative design. Some examples are shown in Fig. 12.

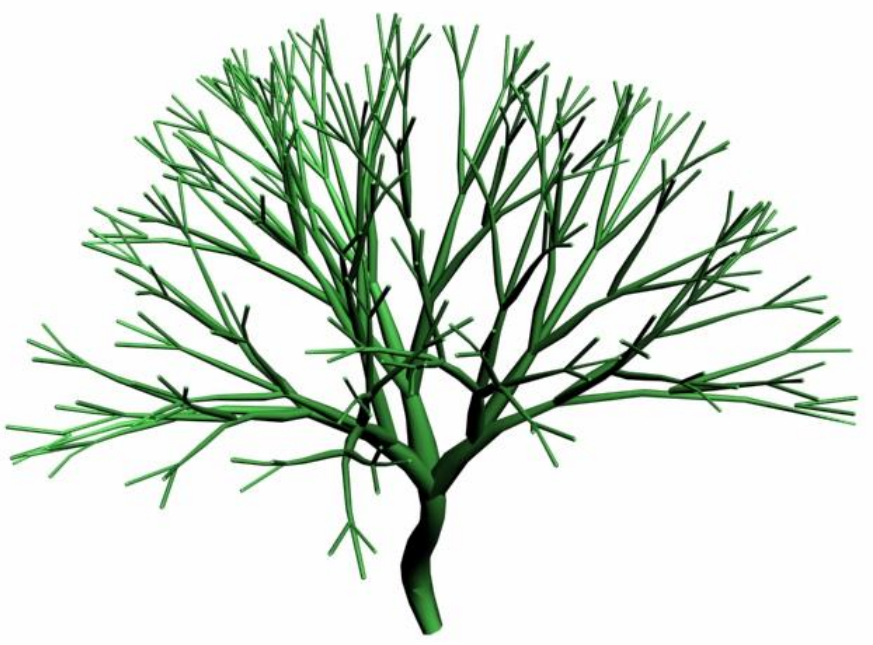

(a) Banyan tree without leaf 


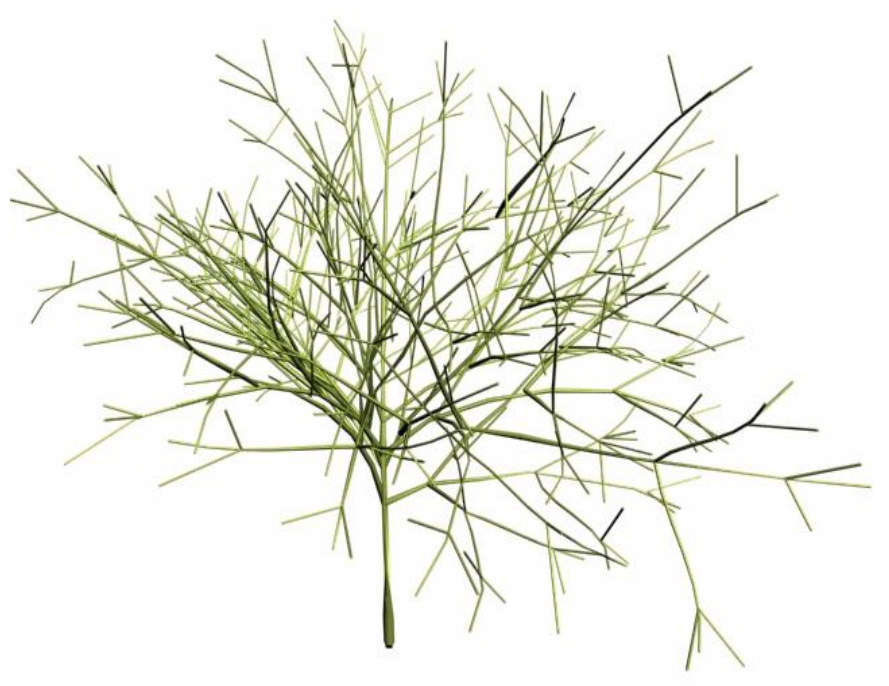

(b) Maple without leaf

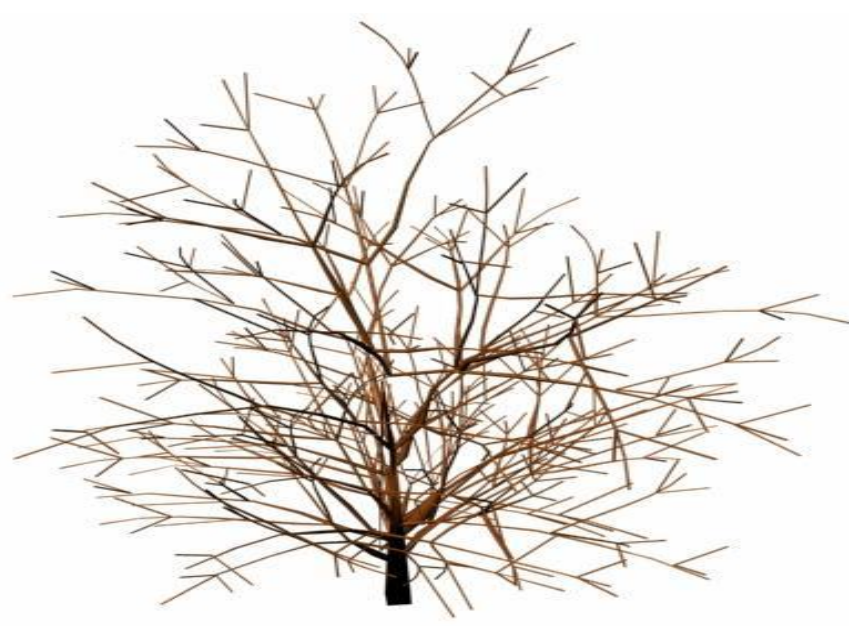

(c) Cherry bay without leaf

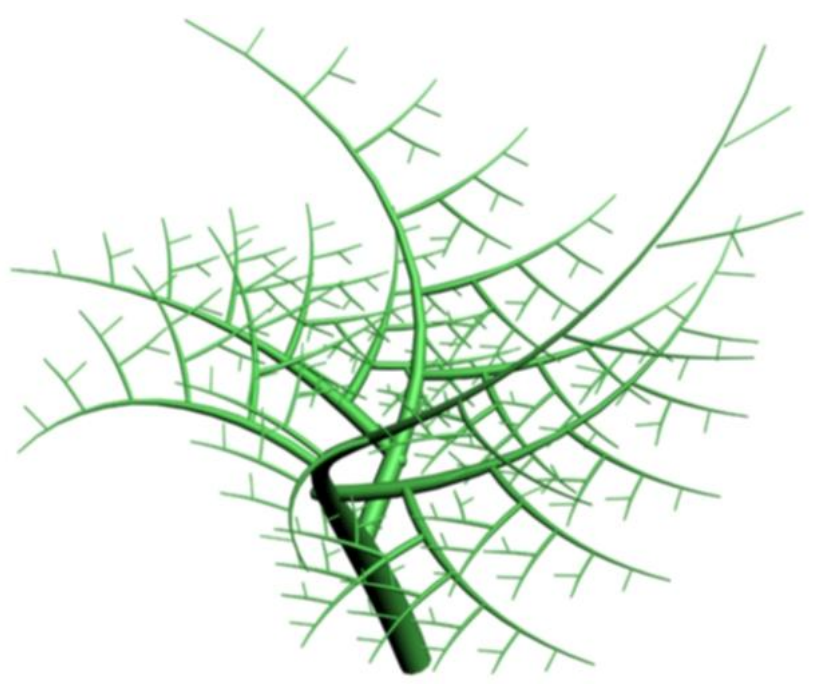

(d) Mighty Oak Tree

Fig. 12 Some examples of modeling tree with BBSCs
The technique can be applied to interactive design of bonsai tree models. A prototype system is developed based on the work [21]. Currently only tree trunks are investigated and our current prototype system is focus on the tree. But these methods can be applied to the interactive modeling of leafs, root systems and flowers. It can also be used to simulate plant growth. Furthermore, some fundamental algorithms like blending between 2 stems, etc. are needed to investigate. As BBSCs are used to represent geometric information of trees, the dataset of the representation is compressed and suitable for transmitting via the internet. So the above methods can be applied to collaborative design of plants in distributed environment. These problems are needed to be investigated in the future.

\section{ACKNOWLEDGEMENT}

We wish to express our appreciations to Miss Xuefeng Ao for her contributions to the development of the interface of our prototype system. I'd like to thank these reviewers for their meaningful suggestions and hard work. The work is partly supported by the National High Technology Research and Development Program of China (863 Program.No: 2008AA01Z301)

\section{REFERENCES}

[1] Long Quan, Ping Tan, Gang Zeng, Lu Yuan, Jingdong Wang and Sing-Bing Kang. "Image-based Plant Modeling". ACM Transactions on Graphics, (TOG) Vol. 25, No. 3. (July 2006), pp. 599-604.

[2] J. Bloomenthal. Modeling the mighty maple. Proceedings of SIGGRAPH '85 (San Francisco, California, July 22-26, 1985) in Computer Graphics, 19, 3 (July 1985), pp 305-311, ACM SIGGRAPH, New York, 1985

[3] N. Greene. Voxel Space Automata: Modeling With Stochastic Growth Processes In Voxel Space. Computer Graphics, July 1989. pp. 175-184

[4] P. Prusinkiewicz and A. Lindenmayer. The Algorithmic Beauty of Plants, Springer Verlag, 1990

[5] C. Galbraith, P. MacMurchy and B. Wyvill. BlobTree Trees. Computer Graphics International 2004, pp. 78-85

[6] R. F. Tobler, S. Maierhofer and A. Wilkie. A Multiresolution Mesh Generation Approach for Procedural Definition of Complex Geometry. Shape Modeling International 2002, pp. 35-42

[7] H. S. Seah and Z. Wu. Ball B-Spline Based Geometric Models in Distributed Virtual Environments. In Workshop towards Semantic Virtual Environments, SVE, Villars, Switzerland, Mar 2005, pp. 1-8.

[8] S. Demko, L. Hodges and B. Naylor. Construction of fractal objects with iterated function systems, ACM SIGGRAPH Computer Graphics, v.19 n.3, pp271-278, Jul. 1985

[9] M. F. Barnsley. Fractals Everywhere, 2nd Edition, Academic Press, New York, 1993.

[10] D. Strnad and N. Guid. Modeling Trees with Hypertextures. Comput. Graph. Forum 23(2): 173-188 (2004)

[11] Hock Soon Seah, Zhongke Wu, Feng Tian, Xuangxiang Xie, Yixiang Lu, Xian Xiao. Modeling and Visualization of Plants with Ball B-Splines. The International Workshop on Advanced Image Technology (IWAIT) 2007, Bangkok, Thailand.

[12] B. Lintermann and O. Deussen. Interactive Modeling of Plants. IEEE Computer and Application, 19(1):2 - 11, 1999.

[13] Okabe, M., Owada, S., and Igarashi, T. Interactive Design of Botanical Trees Using Freehand Sketches and Example-based Editing. Computer Graphics Forum, 24, 3, C487-C496.

[14] Lisa Streit, Paul Lapides, Mario Costa Sousa, Ehud Sharlin. Modeling Plant Variations through 3D Interactive Sketches. 3rd Eurographics Workshop on Sketch-based Interfaces and Modeling Vienna, Austria, August 2, 2006. 
[15] Zhao X., De Reffye P., Barthelemy D., Hu B.-G. Interactive Simulation of Plant Architecture Based on a Dual-Scale Automaton Model. PMA03 Proceedings, Beijing, Oct 13-16, 2003, pp 144-153.

[16] Xi Wang, Lifeng Wang, Ligang Liu, Shimin Hu and Baining Guo. Interactive modeling of tree bark. Proceedings of Pacific Graphics'2003, IEEE Computer Society, Alberta, Canada, Oct, 2003, pp. 83-90.

[17] R.F. Tobler, S. Maierhofer, and A. Wilkie. Mesh-based Parametrized L-Systems and Generalized Subdivision for Generating Complex Geometry. International Journal of Shape Modeling, 8(2):173-191, Dec 2002.

[18] N. Max. Cone-sphere. ACM Transactions on Graphics (SIGGRAPH 1990 Conference Proceedings), 24(2), 1990

[19] J. C. Hart and B. Baker. Implicit modeling of tree surfaces. In Implicit Surfaces'96, pages 143-152, 1996.

[20] C.-L. Jin, X. ans Tai, J. Feng, and Q. Peng. Convolution surfaces for line skeletons with polynomial weight distributions. Journa of Graphics Tools, 6(3):1728, 2001.

[21] W. T. Reeves. Approximate and Probabilistic Algorithms for Shading and Rendering Structured Particle Systems. Computer Graphics, vol. 19, no. 3, pp. 313-322, 1985.

[22] C. Galbraith, L. Mündermann and B. Wyvill. Implicit Visualization and Inverse Modeling of Growing Trees. Comput. Graph. Forum 23(3): 351-360 (2004)

[23] Zhongke Wu, Mingquan Zhou, Xingce Wang, Xuefeng Ao and Rongqing Song. An Interactive System of Modeling Trees with Ball B-Splines. The Second International Symposium on Plant Growth Modeling, Simulation, Visualization and Applications (PMA06). Beijing (CHINA PR), November 13-17, 2006

[24] T. W. Sederberg and R. T. Farouki. Approximation by interval Bézier curves. IEEE Comput. Graph. Appl. 12(5):87-95, 1992.

[25] Qun Lin, Jon G. Rokne: Disk Bézier curves. Computer Aided Geometric Design 15(7): 721-737 (1998)

[26] Germain E. Randriambelosoa. Space Curves Approximation using G1 Ball-Spline Curves, AMRX 5:153-167, 2004.

[27] M. Zerroug and R. Nevatia. Three-dimensional descriptions based on the analysis of the invariant and quai-invariant properties of some curved-axis generalized cylinders. IEEE Transactions on Pattern Analysis and Mathine Intelligence, 18(3):237-253, 1996.

[28] Bronsvoort, W. F. and Waarts, J. J., A method for converting the surface of a generalized cylinder into a B-Spline surface. Computers \& Graphics, 1992, 16(2),175-178.

[29] U. Shani, D.H. Ballard, Splines as Embeddings for Generalized Cylinders, Comput. Vision Graphics Image Process. 27, (1984) 129-156.

[30] Oppenheimer, P. E. Real Time Design and Animation of Fractal Plants and Trees, in Proc. ACM SIGGRAPH, (Dallas, 1986) ACM pp. 55 - 64.

[31] XG Viennot, G Eyrolles, N Janey and D Arques, Combinatorial analysis of ramified patterns and computer imagery of trees. Comput Graph 23 (1989), pp. 31-40.

[32] P. Reffye, C. Edelin, J. Francon, M. Jaeger, and C. Puech, "Plant models faithful to botanical structure and development," Comput. Graph., vol.22, no.4, pp.151-158, Aug. 1988.

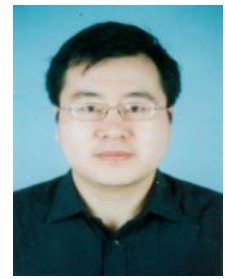

Dr. Zhongke Wu is a professor in college of information science and technology, Beijing Normal University, P.R.China. He received B.Sc in Mathematics from Peking University in China in 1988, and M.Eng and $\mathrm{PhD}$ in CAD/CAM from Beijing University of Aeronautics \& Astronautics, China, in 1991 and 1995 respectively.

His current research interests include computer graphics, geometric modeling, $\mathrm{CAD} / \mathrm{CAM}$, volume graphics and medical imaging, scientific visualization, animation and virtual reality.

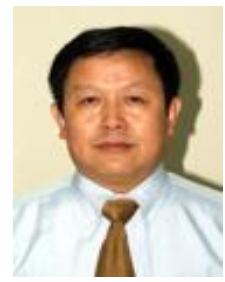

Prof. Mingquan Zhou is the supervisor of doctor candidates and the dean in college of information science and technology, Beijing Normal University, P.R.China. His current research interests include computer graphics, $3 \mathrm{D}$ visualization. He has received 11 awards of province and ministry. He has published over 300 important papers in this field.

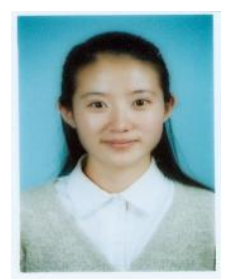

Dr. Xingce Wang is an associate professor in college of information science and technology, Beijing Normal University, P.R.China. She is major in the 3D modeling and $3 \mathrm{D}$ visualization. She current research interest include computer graphic, medical imaging, artificial intelligence and Machine learning. 